Bull. Korean Math. Soc. 46 (2009), No. 6, pp. 1237-1248

DOI 10.4134/BKMS.2009.46.6.1237

\title{
ESTIMATING THE DOMAIN OF ATTRACTION VIA MOMENT MATRICES
}

\author{
Chunji Li, Cheon Seoung Ryoo, Ning Li, and Lili Cao
}

\begin{abstract}
The domain of attraction of a nonlinear differential equations is the region of initial points of solution tending to the equilibrium points of the systems as the time going. Determining the domain of attraction is one of the most important problems to investigate nonlinear dynamical systems. In this article, we first present two algorithms to determine the domain of attraction by using the moment matrices. In addition, as an application we consider a class of SIRS infection model and discuss asymptotical stability by Lyapunov method, and also estimate the domain of attraction by using the algorithms.
\end{abstract}

\section{Introduction}

Estimating the domain of attraction (DOA) of a dynamical system is an important subject of the theory of stability and is well known in the area of nonlinear system analysis and control. The DOA of the system makes an important role in applied mathematics such as electric systems, chemical reactors, and many non-linear dynamical systems, for the work in security. Note that infectious disease is a very common phenomenon. The DOA of this system can be applied to determine and forecast the development trend of infection.

Given the autonomous system

$$
\dot{x}=f(x), \quad x \in \mathbb{R}^{n},
$$

with $f(0)=0$, the domain of attraction (DOA) of $x=0$ is

$$
S=\left\{x^{0} \in \mathbb{R}^{n} \mid \lim _{t \rightarrow \infty} x\left(t, x^{0}\right)=0\right\},
$$

where $x\left(\cdot, x^{0}\right)$ denotes the solution of (1.1) corresponding to the initial condition $x(0)=x^{0}$. Let $V(x)$ be a continuously differentiable real-valued function defined on a domain $D \subset \mathbb{R}^{n}$ containing the origin. The function $V(x)$ is called a Lyapunov function for the system (1.1) if $V(x)$ is positive definite on $D$ and

Received October 24, 2008.

2000 Mathematics Subject Classification. 37C75, 44A60.

Key words and phrases. domain of attraction, moment matrices, Lyapunov function, SIRS model.

(C)2009 The Korean Mathematical Society 
$\dot{V}(x)=\left(\frac{\partial V}{\partial x}\right)^{\mathrm{T}} f(x)$ is negative semidefinite on $D$. In [2] O. Hachicho showed that if the domain

$$
\Omega_{c}=\left\{x \in \mathbb{R}^{n} \mid V(x) \leq c\right\}, \quad c>0,
$$

is bounded, $0 \in \Omega_{c}$ and $\dot{V}(x)$ is negative definite in $\Omega_{c}$, then $\Omega_{c} \subset S$. Let

$$
V(x)=x^{\mathrm{T}} P x, \quad P=P^{\mathrm{T}} \in \mathbb{R}^{n \times n},
$$

where $P$ is a positive definite matrix. The hypersurfaces given by $\dot{V}(x)=0, x \neq$ 0 define the boundary of the region of negative definiteness of $\dot{V}(x)$ in which we seek the guaranteed estimation $\Omega_{c}$. In the case of quadratic Lyapunov functions such an estimation is the interior of the ellipsoid defined by (1.3). Our objective is to find the maximum value $c^{*}$ of $c$ such that $\dot{V}(x)$ is negative definite in $\Omega_{c}$. Note that this $c^{*}$ is defined by the following optimization problem

$$
\left\{\begin{array}{l}
\text { find } c^{*}=\min V(x) \\
\text { subject to the constraints: } \\
\dot{V}(x)=0, \quad x \neq 0 .
\end{array}\right.
$$

This paper is organized as follows. In Section 2 we recall some results from the mathematical theory of moments proved by J. Lasserre in [7], which will be used frequently in this note, and obtain the algorithms that can be used to estimate the DOA for polynomial dynamical systems using polynomial Lyapunov function. As an application, in Section 3, we estimate the domain of attraction of a class of SIRS infection model by using the algorithms. All of the calculations in this paper were obtained throughout computer experiments using the YALMIP-an LMI package of MATLAB ([10]).

\section{The estimation of the DOA via moment matrices}

\subsection{Minimization of polynomials and the problem of moments}

In [7] J. Lasserre considered the following two classical problems.

The problem of global minimization

$$
\mathbb{P} \mapsto p^{*}:=\min _{x \in \mathbb{R}^{n}} p(x) .
$$

The problem of constrained minimization

$$
\mathbb{P}_{K} \mapsto p_{K}^{*}:=\min _{x \in K} p(x),
$$

where $p(x)$ is a real-valued polynomial and $K$ is a compact set defined by polynomial inequalities

$$
g_{i}(x) \geq 0, \quad i=1, \ldots, r .
$$

Let

$$
1, x_{1}, x_{2}, \ldots, x_{n}, x_{1}^{2}, x_{1} x_{2}, \ldots, x_{1} x_{n}, x_{2}^{2}, x_{2} x_{3}, \ldots, x_{1}^{m}, \ldots, x_{n}^{m}
$$


be a basis for the $m$-degree real-valued polynomials $p(x)$ and let $s(2 m)$ be its dimension, where $s(m):=\left(\begin{array}{c}n+m \\ n\end{array}\right)=\frac{(n+m) !}{n ! m !}$. Let

$$
p(x)=\sum_{\alpha} p_{\alpha} x^{\alpha}, \text { with } x^{\alpha}:=x_{1}^{\alpha_{1}} x_{2}^{\alpha_{2}} \cdots x_{n}^{\alpha_{n}} \quad \text { and } \quad \sum_{i=1}^{n} \alpha_{i} \leq m,
$$

where $p=\left\{p_{\alpha}\right\} \in \mathbb{R}^{s(m)}$ is the coefficient vector of $p(x)$ in the basis (2.3).

Given an $s(2 m)$-vector $y:=\left\{y_{\alpha}\right\}$ with first element $y_{0, \ldots, 0}=1$, let $M_{m}(y)$ be the moment matrix of dimension $s(m)$. To illustrate $M_{m}(y)$, let us consider a simple example where $n=2$. In this case the matrix $M_{m}(y)$ is a block matrix

$$
\left\{M_{i, j}(y)\right\}_{0 \leq i, j \leq m}
$$

with

$$
M_{i, j}(y)=\left(\begin{array}{cccc}
y_{i+j, 0} & y_{i+j-1,1} & \cdots & y_{i, j} \\
y_{i+j-1,1} & y_{i+j-2,2} & \cdots & y_{i-1, j+1} \\
\vdots & \vdots & \ddots & \vdots \\
y_{j, i} & y_{j-1, i+1} & \cdots & y_{0, i+j}
\end{array}\right)
$$

such that $y_{i, j}$ represents the $(i+j)$ order moment

$$
y_{i, j}=\int x^{i} y^{j} \mu(d(x, y))
$$

for some probability measure $\mu$ (cf. [1], [5], [8], [9]). In particular, for the case $n=2, m=2$, one obtains

$$
\begin{aligned}
M_{2}(y)= & \left(\begin{array}{cccccc}
M_{0,0}(y) & M_{0,1}(y) & M_{0,2}(y) \\
M_{1,0}(y) & M_{1,1}(y) & M_{1,2}(y) \\
M_{2,0}(y) & M_{2,1}(y) & M_{2,2}(y)
\end{array}\right) \\
= & \left(\begin{array}{c|ccc|ccc}
1 & \mid & y_{1,0} & y_{0,1} & y_{2,0} & y_{1,1} & y_{0,2} \\
- & - & - & - & - & - \\
y_{1,0} & \mid & y_{2,0} & y_{1,1} & y_{3,0} & y_{2,1} & y_{1,2} \\
y_{0,1} & y_{1,1} & y_{0,2} & y_{2,1} & y_{1,2} & y_{0,3} \\
- & - & - & - & - & - \\
y_{2,0} & \mid & y_{3,0} & y_{2,1} & y_{4,0} & y_{3,1} & y_{2,2} \\
y_{1,1} & y_{2,1} & y_{1,2} & y_{3,1} & y_{2,2} & y_{1,3} \\
y_{0,2} & y_{1,2} & y_{0,3} & y_{2,2} & y_{1,3} & y_{0,4}
\end{array}\right) .
\end{aligned}
$$

It is not difficult to conclude that for any $N \in \mathbb{N}$ we have

$$
M_{N}(y)=M_{N}^{\mathrm{T}}(y) \quad \text { and } \quad M_{N}(y) \in \mathbb{R}^{s(N) \times s(N)} .
$$

Let $g(x): \mathbb{R}^{n} \rightarrow \mathbb{R}$ be a real valued polynomial of degree $w$ with coefficient vector $g \in \mathbb{R}^{s(w)}$. For the $(i, j)$ entry $y_{\beta}$ of the matrix $M_{m}(y)$, we write $\beta(i, j)$ for the subscript $\beta$ of $y_{\beta}$. Then $M_{m}(g y)$ is defined by

$$
M_{m}(g y)(i, j)=\sum_{\alpha} g_{\alpha} y_{\{\beta(i, j)+\alpha\}}
$$


Let $\operatorname{deg} g_{i}(x)=w_{i}$ and define

$$
\tilde{w}_{i}=\left\lceil\frac{w_{i}}{2}\right\rceil
$$

which is the smallest integer larger than $\frac{w_{i}}{2}$. Then optimization problem $(2.2)$ is equivalent to the following problem

$$
\mathbb{Q}_{K}^{N} \mapsto\left\{\begin{array}{l}
\inf _{y} \sum_{\alpha} p_{\alpha} y_{\alpha} \\
\text { subject to the constraints: } \\
M_{N}(y) \geq 0, \\
M_{N-\tilde{w}_{i}}\left(g_{i} y\right) \geq 0, i=1, \ldots r .
\end{array}\right.
$$

The number $N$ has to be chosen according to the following conditions

$$
N \geq\left\lceil\frac{m}{2}\right\rceil \text { and } N \geq \max _{i} \tilde{w}_{i}
$$

J. Lasserre also formulated that $\left(\mathbb{Q}_{K}^{N}\right)^{*}$, the dual of $\mathbb{Q}_{K}^{N}$,

$$
\left(\mathbb{Q}_{K}^{N}\right)^{*} \mapsto\left\{\begin{array}{l}
\inf _{X_{i}, Z_{i}} X(1,1)+\sum_{i=1}^{r} g_{i}(0) Z_{i}(1,1), \\
\text { subject to the constraints: } \\
\left\langle X, B_{\alpha}\right\rangle+\sum_{i=1}^{r}\left\langle Z_{i}, C_{i \alpha}\right\rangle=p_{\alpha}, \alpha \neq 0 \\
X, Z_{i} \geq 0, i=1, \ldots, r
\end{array}\right.
$$

where $\langle\cdot, \cdot\rangle$ means the trace inner product, that is, $\langle A, B\rangle=\operatorname{tr}(A B)$. The matrices $B_{\alpha}$ and $C_{i \alpha}$ are derived from $M_{N}(y)$ and $M_{N-\tilde{w}}\left(g_{i} y\right)$ as follow

$$
M_{N}(y)=B_{0}+\sum_{\alpha \neq 0}^{\alpha} B_{\alpha} y_{\alpha}, \quad M_{N-\tilde{w}}\left(g_{i} y\right)=\sum_{\alpha} C_{i \alpha} y_{\alpha}
$$

The following theorem will be used crucially for our work.

Theorem 2.1 ([7]). Let $K \subseteq\{x:\|x\| \leq a\}$ for sufficiently large $a>0$. Then $\inf \mathbb{Q}_{K}^{N} \uparrow p_{K}^{*}$ as $N \rightarrow \infty$. If $K$ has nonempty interior for $N$ sufficient large, then there is no duality gap between $\mathbb{Q}_{K}^{N}$ and its dual $\left(\mathbb{Q}_{K}^{N}\right)^{*}$.

\subsection{Algorithms for estimations}

We first modify algorithms in [3] as following.

\section{Algorithm 2.2 (The primal problem).}

I. Rewrite $\Omega_{c}$ in $(1.3)$ as $\Omega_{c}=\left\{x \mid V(x) \leq c_{0}\right\} \cup\left\{x \mid c_{0} \leq V(x) \leq c\right\}$ such that $\dot{V}(x)<0$ in $\Omega_{c_{0}} \backslash\{0\}$.

II. Rewrite (1.5) as

$$
\left\{\begin{array}{l}
\text { find } c^{*}=\min V(x) \\
\text { subject to the constraints: } \\
g_{1}(x)=\dot{V}(x) \geq 0 \\
g_{2}(x)=x^{\mathrm{T}} x-c_{0} \geq 0, \\
g_{3}(x)=-x^{\mathrm{T}} x+R \geq 0 .
\end{array}\right.
$$


III. Translation (2.9) to (2.6).

IV. Use an LMI-solver to compute $c^{*}$.

Algorithm 2.3 (The dual problem).

I. Define block diagonal matrix $T_{S}:=\operatorname{Diag}\left\{\left(T_{S}\right)_{1,1},\left(T_{S}\right)_{2,2}, \ldots,\left(T_{S}\right)_{r+1, r+1}\right\}$, where

$$
\left(T_{S}\right)_{1,1}=\left(\begin{array}{cc}
1 & \\
& 0
\end{array}\right), \text { and }\left(T_{S}\right)_{i, i}=\left(\begin{array}{cc}
g_{i}(0) & \\
& 0
\end{array}\right), \quad i=2, \ldots, r+1 .
$$

II. Define $X_{S}:=\operatorname{Diag}\left\{X, Z_{1}, \ldots, Z_{r}\right\}$.

III. Provide all $\alpha \neq 0, \ldots, 0$ with an index $k$, and define $b_{k}=p_{\alpha_{k}},\left(\alpha_{k} \neq\right.$ $0, \ldots, 0)$.

IV. Define $\left(A_{S}\right)_{k}:=\operatorname{Diag}\left\{B_{\alpha_{k}}, C_{1 \alpha_{k}}, C_{2 \alpha_{k}}, \ldots, C_{r \alpha_{k}}\right\}$.

V. Transform the dual problem (2.7) into the following semidefinite problem:

$$
\left\{\begin{array}{l}
\min \left\langle T_{S}, X_{S}\right\rangle \\
\text { subject to the constraints: } \\
\left\langle\left(A_{S}\right)_{k}, X_{S}\right\rangle=b_{k}, k=1, \ldots, l, \\
X_{S} \geq 0 .
\end{array}\right.
$$

VI. Solve optimization problem (2.10), by using the LMI-solver toolbox, such as YALMIP ([10]).

By using the algorithms, we can obtain the following result.

Theorem 2.4. Let $V=x_{1}^{2}+x_{2}^{2}$ be the Lyapunov function for the following 2-dimensional nonlinear system

$$
\left\{\begin{array}{l}
\frac{d x_{1}}{d t}=-x_{1} \\
\frac{d x_{2}}{d t}=-x_{2}+x_{1}^{2} x_{2}
\end{array}\right.
$$

Then $\Omega_{c}=\left\{\left(x_{1}, x_{2}\right) \in \mathbb{R}^{2} \mid x_{1}^{2}+x_{2}^{2} \leq 4\right\}$ is a subset of the domain of attraction for system (2.11).

Proof. By direct calculation, we have $\dot{V}=-2 x_{1}^{2}-x_{2}^{2}+2 x_{1}^{2} x_{2}^{2}$. First, by Algorithm 2.2 , we need to solve the following optimization problem

$$
\left\{\begin{array}{l}
\text { find } c^{*}=\min \left(x_{1}^{2}+x_{2}^{2}\right) \\
\text { subject to the constraints: } \\
g_{1}(x)=-2 x_{1}^{2}-x_{2}^{2}+2 x_{1}^{2} x_{2}^{2} \geq 0, \\
g_{2}(x)=x_{1}^{2}+x_{2}^{2}-c_{0} \geq 0 \\
g_{3}(x)=-x_{1}^{2}-x_{2}^{2}+R \geq 0 .
\end{array}\right.
$$

And rewrite (2.12) as

$$
\left\{\begin{array}{l}
\inf _{y} \sum_{\alpha} p_{\alpha} y_{\alpha}=y_{2,0}+y_{0,2} \\
\text { subject to the constraints: } \\
M_{N}(y) \geq 0 \\
M_{N-\tilde{w}_{i}}\left(g_{i} y\right) \geq 0, \quad i=1,2,3 .
\end{array}\right.
$$




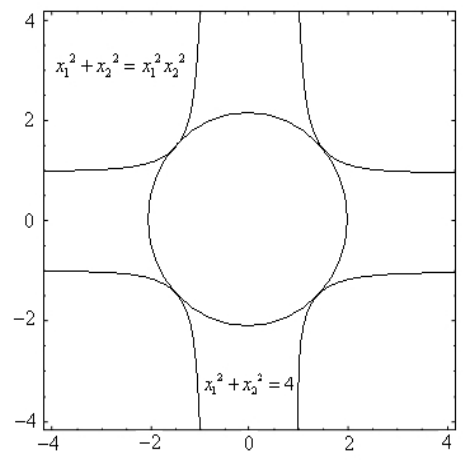

Figure 1. The subset of the domain of attraction for system (2.11)

If we choose $N=4, c_{0}=1, R=5$ and use an LMI-solver, then we can obtain $c^{*}=4$. Second, by Algorithm 2.3, we need to solve the optimization problem (2.10). Here $b_{0}=b_{1}=1$, and

$$
T_{S}=\operatorname{Diag}\left(\left(T_{S}\right)_{1,1},\left(T_{S}\right)_{2,2},\left(T_{S}\right)_{3,3},\left(T_{S}\right)_{4,4}\right),
$$

where

$$
\begin{aligned}
& \left(T_{S}\right)_{1,1}=\left(\begin{array}{ll}
1 & 0 \\
0 & 0
\end{array}\right),\left(T_{S}\right)_{2,2}=\left(\begin{array}{ll}
0 & 0 \\
0 & 0
\end{array}\right), \\
& \left(T_{S}\right)_{3,3}=\left(\begin{array}{cc}
-c_{0} & 0 \\
0 & 0
\end{array}\right),\left(T_{S}\right)_{4,4}=\left(\begin{array}{cc}
R & 0 \\
0 & 0
\end{array}\right),
\end{aligned}
$$

and

$$
\begin{aligned}
X_{S} & =\operatorname{Diag}\left(X, Z_{1}, Z_{2}, Z_{3}\right), \\
\left(A_{S}\right)_{1} & =\operatorname{Diag}\left(B_{2,0}, C_{1,2,0}, C_{2,2,0}, C_{3,2,0}\right), \\
\left(A_{S}\right)_{2} & =\operatorname{Diag}\left(B_{0,2}, C_{1,0,2}, C_{2,0,2}, C_{3,0,2}\right) .
\end{aligned}
$$

If we choose $N=4, c_{0}=1, R=5$, and use an LMI-solver, we can also obtain $c^{*}=4$.

Remark. Figure 1 represents the subset of the domain of attraction for system (2.11).

\section{The DOA of SIRS epidemic model}

In recent years, many researches studied SIRS epidemic model (see [4], [6]). In this section, we consider the following SIRS model

$$
\left\{\begin{array}{l}
\frac{d S}{d t}=A-\beta S I-d S+c I+\delta R=f, \\
\frac{d I}{d t}=\beta S I-r I-d I-\alpha I-c I=g, \\
\frac{d R}{d t}=r I-d R-\delta R=h,
\end{array}\right.
$$


where $S(t)$ is the number of susceptible individuals at time $t, I(t)$ is the number of infective individuals at time $t, R(t)$ is the number of recovered individuals at time $t, \beta$ is the infection rate, $A$ is the recruitment rate of the population, $c$ is the sensible rate without immunity, $d$ is the natural mortality rate of the population, $r$ is the recovery rate of infective individuals, $\alpha$ is the death rate due to disease, and $\delta$ is the rate that removed return to the susceptible class. $N=S+I+R$ is the number of the total population. And $R_{0}=\frac{\beta A}{d(d+\alpha+r+c)}$ denotes the basic reproduction number.

Let $P_{1}\left(\frac{A}{d}, 0,0\right)$ and $P_{2}\left(\frac{d+\alpha+r+c}{\beta}, \frac{\left(A \beta-c d-d \alpha-d r-d^{2}\right)(d+\delta)}{\beta\left(d \alpha+d r+d \delta+\alpha \delta+d^{2}\right)}, \frac{\left(A \beta-c d-d \alpha-d r-d^{2}\right) r}{\beta\left(d \alpha+d r+d \delta+\alpha \delta+d^{2}\right)}\right)$. First we give the following theorem.

Theorem 3.1. If $R_{0}<1$, then $P_{1}$ is the unique equilibrium point of (3.1), and it is globally asymptotically stable; if $R_{0}>1$, then $P_{1}$ and $P_{2}$ are two equilibrium points of (3.1), which $P_{1}$ is unstable, but $P_{2}$ is locally asymptotically stable.

Proof. Let

$$
M:=\left(\begin{array}{ccc}
\frac{\partial f}{\partial S} & \frac{\partial f}{\partial I} & \frac{\partial f}{\partial R} \\
\frac{\partial g}{\partial S} & \frac{\partial g}{\partial \hbar} & \frac{\partial g}{\partial \hbar} \\
\frac{\partial h}{\partial S} & \frac{\partial h}{\partial I} & \frac{\partial \hbar}{\partial R}
\end{array}\right)=\left(\begin{array}{ccc}
-d-I \beta & c-S \beta & \delta \\
I \beta & S \beta-d-r-\alpha-c & 0 \\
0 & r & -d-\delta
\end{array}\right) .
$$

For $P_{1}$, we have

$$
\left.M\right|_{P_{1}}=M_{1}=\left(\begin{array}{ccc}
-d & c-\frac{A}{d} \beta & \delta \\
0 & \frac{A}{d} \beta-d-r-\alpha-c & 0 \\
0 & r & -d-\delta
\end{array}\right)
$$

the eigenvalues of $M_{1}$ are

$$
\begin{aligned}
& \lambda_{1}=-d<0 \\
& \lambda_{2}=-d-\delta<0 \\
& \lambda_{3}=\frac{1}{d}\left(-c d-d r+A \beta-d \alpha-d^{2}\right) .
\end{aligned}
$$

So, when $R_{0}=\frac{\beta A}{d(d+\alpha+r+c)}<1$, we know that $\lambda_{3}<0$, and thus $P_{1}$ is locally asymptotically stable. Since $P_{1}$ is unique, we can also know that $P_{1}$ is globally asymptotically stable. When $R_{0}=\frac{\beta A}{d(d+\alpha+r+c)}>1$, we know that $\lambda_{3}>0$, and thus $P_{1}$ is unstable.

For $P_{2}$, we have

$$
\left.M\right|_{P_{2}}=M_{2}=\left(\begin{array}{ccc}
-d+\frac{\left(c d-A \beta+d \alpha+d r+d^{2}\right)(d+\delta)}{\left(d \alpha+d r+d \delta+\alpha \delta+d^{2}\right)} & -d-\alpha-r & \delta \\
-\frac{\left(c d-A \beta+d \alpha+d r+d^{2}\right)(d+\delta)}{\left(d \alpha+d r+d \delta+\alpha \delta+d^{2}\right)} & 0 & 0 \\
0 & r & -d-\delta
\end{array}\right),
$$

and the characteristic polynomial is

$$
X^{3}+a_{1} X^{2}+a_{2} X+a_{3}
$$


with

$$
\begin{aligned}
& a_{1}=2 d+\delta+\frac{(d+\delta)\left(A \beta-c d-d r-d \alpha-d^{2}\right)}{d r+d \alpha+d \delta+\alpha \delta+d^{2}}, \\
& a_{2}=-\frac{\Theta(d+\delta)}{d r+d \alpha+d \delta+\alpha \delta+d^{2}}, \\
& a_{3}=\left(A \beta-c d-d r-d \alpha-d^{2}\right)(d+\delta)>0,
\end{aligned}
$$

where

$$
\begin{aligned}
\Theta= & d^{3}+2 d^{2}(c+r+\alpha)-\beta A(r+\alpha+\delta) \\
& +d\left(c r-2 A \beta+c \alpha+c \delta+2 r \alpha+r \delta+r^{2}+\alpha^{2}\right) .
\end{aligned}
$$

It is easy to show that $a_{1}>0$. Furthermore, $\operatorname{det} \Delta_{2}=\left|\begin{array}{cc}a_{1} & 1 \\ a_{3} & a_{2}\end{array}\right|>0$. In fact, let $\Omega=A \beta-\left(d r+d \alpha+d c+d^{2}\right)>0$. Then we have

$$
\operatorname{det} \Delta_{2}=\frac{\Lambda}{\left(d r+d \alpha+d \delta+\alpha \delta+d^{2}\right)^{2}},
$$

where

$$
\begin{aligned}
\Lambda= & \Omega^{2}(d+\delta)^{2}(2 d+r+\alpha+\delta) \\
& +\Omega(d+\delta)\left(d r+d \alpha+4 d \delta+r \delta+4 d^{2}+\delta^{2}\right)\left(d r+d \alpha+d \delta+\alpha \delta+d^{2}\right) \\
& +d(d+\delta)(2 d+\delta)\left(d r+d \alpha+d \delta+\alpha \delta+d^{2}\right)^{2} .
\end{aligned}
$$

Hence $P_{2}$ is locally asymptotically stable, when $R_{0}>1$.

Notice that $P_{2}$ is local asymptotically stable, when $R_{0}>1$. We take $A=$ $4, \alpha=\beta=r=d=\delta=c=\frac{1}{2}$. Then $R_{0}=\frac{\beta A}{d(d+\alpha+r+c)}=2>1$. And the coordinate of the point $P_{2}$ is $\left(4, \frac{8}{5}, \frac{4}{5}\right)$. Let $S=x+4, I=y+\frac{8}{5}, R=z+\frac{4}{5}$. Then we obtain

$$
\left(\begin{array}{l}
\frac{d x}{d t} \\
\frac{d y}{d t} \\
\frac{d z}{d t}
\end{array}\right)=\left(\begin{array}{ccc}
-\frac{13}{10} & -\frac{3}{2} & \frac{1}{2} \\
\frac{4}{5} & 0 & 0 \\
0 & \frac{1}{2} & -1
\end{array}\right)\left(\begin{array}{l}
x \\
y \\
z
\end{array}\right)+\left(\begin{array}{c}
-\frac{1}{2} x y \\
\frac{1}{2} x y \\
0
\end{array}\right) .
$$

The linearized system of (3.2) is

$$
\left(\begin{array}{l}
\frac{d x}{d t} \\
\frac{d y}{d t} \\
\frac{d z}{d t}
\end{array}\right)=\left(\begin{array}{ccc}
-\frac{13}{10} & -\frac{3}{2} & \frac{1}{2} \\
\frac{4}{5} & 0 & 0 \\
0 & \frac{1}{2} & -1
\end{array}\right)\left(\begin{array}{l}
x \\
y \\
z
\end{array}\right)
$$

Proposition 3.2. The function

$$
V(x, y, z)=\frac{299}{475} x^{2}+\frac{6457}{3800} y^{2}+\frac{286}{475} z^{2}+\frac{378}{475} x y+\frac{184}{475} y z+\frac{194}{475} x z
$$

is a Lyapunov function for system (3.3). 
Proof. Notice that

$$
V(x, y, z)=(x, y, z) M\left(\begin{array}{l}
x \\
y \\
z
\end{array}\right) \quad \text { and } \quad M=\left(\begin{array}{ccc}
\frac{299}{475} & \frac{189}{475} & \frac{97}{475} \\
\frac{189}{475} & \frac{6457}{3800} & \frac{92}{475} \\
\frac{97}{475} & \frac{82}{475} & \frac{286}{475}
\end{array}\right) .
$$

Since $\operatorname{det}[M]_{1}=\frac{299}{475}, \operatorname{det}[M]_{2}=\frac{13159}{14440}$, and $\operatorname{det} M=\frac{35067}{72200}$, we obtain that matrix $M$ is positive definite. And by a simple computation we obtain that

$$
\begin{aligned}
\left.\frac{d V}{d t}\right|_{(3.3)} & =\frac{\partial V}{\partial x} \frac{d x}{d t}+\frac{\partial V}{\partial y} \frac{d y}{d t}+\frac{\partial V}{\partial z} \frac{d z}{d t} \\
& =-x^{2}-y^{2}-z^{2} .
\end{aligned}
$$

Thus we have our conclusion.

Remark. For the Lyapunov function in Proposition 3.2, we have

$$
\left.\frac{d V}{d t}\right|_{(3.2)}=-x^{2}-y^{2}-z^{2}+\frac{989}{760} x y^{2}-\frac{1}{95} x y z-\frac{22}{95} x^{2} y .
$$

Theorem 3.3. The region $\Omega_{c}=S \cap E$ is a subset of the domain of attraction for system (3.2) with

$$
\begin{aligned}
& S=\left\{(x, y, z) \in \mathbb{R}^{3} \mid x \geq-4, y \geq-\frac{8}{5}, z \geq-\frac{4}{5}\right\}, \\
& E=\left\{(x, y, z) \in \mathbb{R}^{3} \mid \varphi(x, y, z) \leq 2.6094\right\},
\end{aligned}
$$

where $\varphi(x, y, z)=\frac{299}{475} x^{2}+\frac{6457}{3800} y^{2}+\frac{286}{475} z^{2}+\frac{378}{475} x y+\frac{184}{475} y z+\frac{194}{475} x z$.

Proof. By Algorithm 2.2, we need to solve the following optimization problem $\left\{\begin{array}{l}\min \left(\frac{299}{475} x^{2}+\frac{6457}{3800} y^{2}+\frac{286}{475} z^{2}+\frac{378}{475} x y+\frac{184}{475} y z+\frac{194}{475} x z\right) \\ \text { such that }\left\{\begin{array}{l}g_{1}(x, y, z)=-x^{2}-y^{2}-z^{2}+\frac{989}{760} x y^{2}-\frac{1}{95} x y z-\frac{22}{95} x^{2} y \geq 0 \\ g_{2}(x, y, z)=\left(x^{2}+y^{2}+z^{2}\right)-1 \geq 0 \\ g_{3}(x, y, z)=4-\left(x^{2}+y^{2}+z^{2}\right) \geq 0 .\end{array}\right.\end{array}\right.$

It is equivalent to solve the following optimization problem (3.4)

$$
\left\{\begin{array}{l}
c^{*}=\min \left(\frac{299}{475} y_{2,0,0}+\frac{6457}{3800} y_{0,2,0}+\frac{286}{475} y_{0,0,2}+\frac{378}{475} y_{1,1,0}+\frac{184}{475} y_{0,1,1}+\frac{194}{475} y_{1,0,1}\right) \\
\text { such that }\left\{\begin{array}{l}
M_{N}(y) \geq 0, \\
M_{N-\tilde{w}_{i}}\left(g_{i} y\right) \geq 0, i=1,2,3 .
\end{array}\right.
\end{array}\right.
$$

Since, $w_{1}=3, w_{2}=2, w_{3}=2, m=2$, and $N \geq \max \tilde{w}_{i}=\max \left\lceil\frac{w_{i}}{2}\right\rceil=2$ and $N \geq\left\lceil\frac{m}{2}\right\rceil=2$, we first take $N=3$. Then

$$
M_{3}(y)=\left(\begin{array}{cccc}
M_{0,0,0} & M_{0,0,1} & M_{0,0,2} & M_{0,0,3} \\
M_{1,0,0} & M_{1,0,1} & M_{1,0,2} & M_{1,0,3} \\
M_{2,0,0} & M_{2,0,1} & M_{2,0,2} & M_{2,0,3} \\
M_{3,0,0} & M_{3,0,1} & M_{3,0,2} & M_{3,0,3}
\end{array}\right),
$$




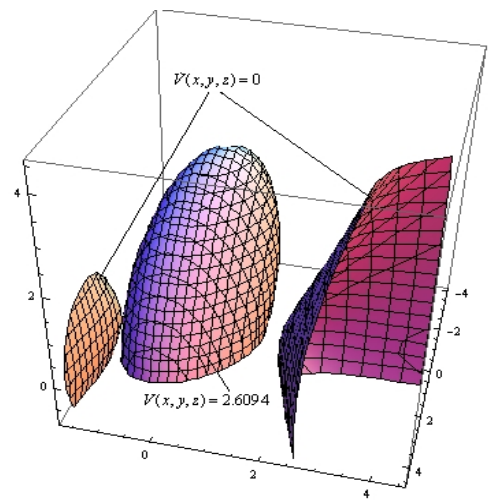

FIGURE 2. The subset of the domain of attraction for SIRS system (3.2)

where

$$
M_{i, j, k}=\left(\begin{array}{ccccc}
y_{i+j+k, 0,0} & y_{i+j+k-1,1,0} & y_{i+j+k-1,0,1} & y_{i+j+k-2,2,0} & \cdots \\
y_{i+j+k-1,1,0} & y_{i+j+k-2,2,0} & y_{i+j+k-2,1,1} & y_{i+j+k-3,3,0} & \cdots \\
y_{i+j+k-1,0,1} & y_{i+j+k-2,1,1} & y_{i+j+k-2,0,2} & y_{i+j+k-3,2,1} & \cdots \\
y_{i+j+k-2,2,0} & y_{i+j+k-3,3,0} & y_{i+j+k-3,2,1} & y_{i+j+k-4,4,0} & \cdots \\
\vdots & \vdots & \vdots & \vdots & \ddots
\end{array}\right),
$$

and

$$
M_{1}\left(g_{r} y\right)=\left(\begin{array}{cccc}
G_{r}(0,0,0) & G_{r}(1,0,0) & G_{r}(0,1,0) & G_{r}(0,0,1) \\
G_{r}(1,0,0) & G_{r}(2,0,0) & G_{r}(1,1,0) & G_{r}(1,0,1) \\
G_{r}(0,1,0) & G_{r}(1,1,0) & G_{r}(0,2,0) & G_{r}(0,1,1) \\
G_{r}(0,0,1) & G_{r}(1,0,1) & G_{r}(0,1,1) & G_{r}(0,0,2)
\end{array}\right), r=1,2,3,
$$

with

$$
\begin{aligned}
G_{1}(i, j, k)= & \frac{989}{760} y_{1+i, 2+j, k}-y_{2+i, j, k}-y_{i, 2+j, k}-y_{i, j, 2+k} \\
& -\frac{1}{95} y_{1+i, 1+j, 1+k}-\frac{22}{95} y_{2+i, 1+j, k}, \\
G_{2}(i, j, k)= & y_{2+i, j, k}+y_{i, 2+j, k}+y_{i, j, 2+k}-y_{i, j, k}, \\
G_{3}(i, j, k)= & -y_{2+i, j, k}-y_{i, 2+j, k}-y_{i, j, 2+k}+4 y_{i, j, k} .
\end{aligned}
$$

By using the YALMIP-yet another LMI package of Matlab (see [10]), we can solve the optimization problem $(3.4)$, and obtain $c^{*} \approx 1.5590$. Similarly, we can consider the cases $N=4$ and $N=5$. Thus we obtain same values $c^{*} \approx 2.6094$. Next, by Algorithm 2.3, we can obtain $c^{*} \approx 1.5843$, and 2.6094, when $N=3$ and 4 , respectively. Therefore, we have our conclusion.

Remark. Figure 2 shows the subset of the domain of attraction for system (3.2). 


\section{References}

[1] R. Curto and L. Fialkow, Solution of the truncated complex moment problem for flat data, Mem. Amer. Math. Soc. 119 (1996), no. 568, x+52 pp.

[2] O. Hachicho, A novel LMI-based optimization algorithm for the guaranteed estimation of the domain of attraction using rational Lyapunov functions, J. Franklin Inst. 344 (2007), no. 5, 535-552.

[3] O. Hachicho and B. Tibken, Estimating domains of attractions of a class of nonlinear dynamical systems with LMI methods based on the theory of moments, Proceedings of the 41st IEEE Conference on Decision and Control, pp. 3150-3155, Las Vegas, USA, 2002.

[4] Y. Jin, W. Wang, and S. Xiao, An SIRS model with a nonlinear incidence rate, Chaos Solitons Fractals 34 (2007), no. 5, 1482-1497.

[5] I. B. Jung, E. Ko, C. Li and, S. S. Park, Embry truncated complex moment problem, Linear Algebra Appl. 375 (2003), 95-114.

[6] X. J. Lan, H. X. Yang, and Z. Q. Sun, Class of the combined SIR and SIS contagion model for population for varying size, J. Chongqing Institute Technology (Natural Science Edition, Chinese) $\mathbf{2 1}$ (2007), no. 6, 40-42.

[7] J. B. Lasserre, Global optimization with polynomials and the problem of moments, SIAM J. Optim. 11 (2001), no. 3, 796-817.

[8] C. Li, I. B. Jung, and S. S. Park, Complex moment matrices via Halmos-Bram and Embry conditions, J. Korean Math. Soc. 44 (2007), no. 4, 949-970.

[9] C. Li and S. H. Lee, The quartic moment problem, J. Korean Math. Soc. 42 (2005), no. $4,723-747$.

[10] J. Lofberg, Yalmip : A toolbox for modeling and optimization in matlab, in Proceedings of the CACSD Conference, Taipei, Taiwan, 2004.

Chunji Li

Institute OF System Science

College of ScIences

NORTHEASTERN UNIVERSITY

Shenyang 110-004, P. R. China

(Current Address)

Department of Mathematics

HANNAM UNIVERSITY

DAeJeOn 306-791, KoreA

E-mail address: chunjili@hanmail.net, chunjili2000@yahoo.com.cn

Cheon Seoung Ryoo

Department of Mathematics

HANNAM UNIVERSITY

DAEJEON 306-791, KOREA

E-mail address: ryoocs@hnu.kr

Ning LI

Institute of System Science

College of Sciences

Northeastern University

Shenyang 110-004, P. R. China

(Current Address)

Shanghai Huawei Technologies Co. Ltd.

Shanghai 201-206, P. R. China

E-mail address: lining_neu@yahoo.com.cn 
Lili CAO

Institute of System Science

College of Sciences

Northeastern University

Shenyang 110-004, P. R. China

(Current Address)

Labor and Social Insurance Bureau

Wenshang 272-500, P. R. China

E-mail address: caolili_5230163.com 